Pensamiento Crítico Vol. 20 № 1, pp. 97-123

\title{
Antecedentes teóricos de la incidencia de la política monetaria en los ciclos económicos
}

Theoretical background of the impact of monetary policy on business

cycles

[Recepción: Marzo de 2015/ Conformidad: Mayo 2015]

Pablo Rivas Santos*

\section{RESUMEN}

El presente artículo, siguiendo a Alonso Neira, Rallo Julián y Philipp Bagus, describe sistemáticamente los antecedentes teóricos de la incidencia de la política monetaria en los ciclos económicos, considerando que los economistas han tratado de explicar porqué la Producción muestra ciclos de duración variable. Esta explicación intenta determinar tres aspectos: 1) Qué tipo de perturbación -exógena o endógena, real o monetaria- golpea a la producción generando ciclos; 2) qué canales de transmisión expanden esa perturbación por todo el sistema económico; y 3) qué factores inciden en la persistencia del ciclo económico, convirtiendo esa perturbación en ciclos de mayor amplitud y resistencia.

Palabras clave: Política Monetaria, Canales de Transmisión, Persistencia, Perturbación Monetaria.

* Profesor de Economía e Investigador del Instituto de Investigaciones Económicas de la Facultad de Ciencias Económicas de la Universidad Nacional Mayor de San Marcos. email: pablorivas_2000@yahoo.es 


\section{Pensamiento Crítico Vol. 20. NoI}

\section{Clasificación JEL: E32}

\section{SUMMARY}

This paper, following Alonso Neira, Rallo Julián y Philipp Bagus, systematically describes the theoretical background of the impact of Monetary Policy on Economic Cycles; where as economists have tried to explain why the production cycles of varying duration shown. This explanation attempts to determine three issues: 1) Whatkind of -exógena or endogenous, real or monetary shock hits the Production generating cycles; what transmission channels expand he disturbance throughout the economic system; and 3) what factors influence the persistence of business cycle turning at temporary disruption in cycles of greater range and endurance.

Keywords: Monetary Policy Transmission Channels, Persistence, Monetary Disturbance

\section{Introducción}

Una visión retrospectiva de las teorías del ciclo económico permite clasificarlas en:

\subsection{Teorías del ciclo exógeno}

Los teóricos del ciclo exógeno atribuyen los ciclos a los efectos acumulativos de schoks aleatorios exógenos repetitivos -schocks tecnológicos, alteraciones drásticas en el precio de las materias primas- que distorsionan el funcionamiento del sistema económico inherentemente estable.

Esta idea de que los ciclos derivande perturbaciones aleatorias exógenas, nace de los modelos estocásticos del ciclo económico de Frisch (1933) y Slutzky (1937). Ambos teóricos demostraron que (bajos ciertas restricciones monetarias) lossistemas dinámicos deecuaciones lineales (con ecuaciones en diferencias para el consumo e inversión) generan ciclos, cuando se someten a perturbaciones exógenas. Estos ciclos tienden a amortiguarse con el paso del tiempo, aunque no desaparecen completamenteporque aparecen nuevas perturbaciones cuyos efectos se suman a los efectos de las anteriores perturbaciones. 


\section{Pablo Rivas Santos}

En estos modelosla longitud de los ciclos y su tendencia a amortiguarse a lo largo del tiempo dependen de los canales de Transmisión de esos sistemas dinámicos de ecuaciones sometido a perturbaciones exógenas; y la intensidad de los ciclos responde a la naturaleza del Impulso Externo. Si bien la teoría Impulso-Transmisión ha sido uno de los principios subyacentes al desarrollo de la teoría neoclásica del ciclo; Hicks (1950) sostiene que no ofrece una explicación satisfactoria de los ciclos observados, ya que se limita a atribuirlas a fuerzas ajenas al propio funcionamiento del sistema económico.

\subsection{Teorías del ciclo endógeno}

Los teóricos del ciclo endógeno (Jhon Maynard Keynes, 1936, Arthur Spiethoff, 1953) atribuyen los ciclos a resultados de la propia dinámica interna del sistema económico. Estos teóricos sostiene que (incluso en ausencia de perturbaciones exógenas) las economías muestran cierta inestabilidad inherente, que se traduce en crisis de sobreproducción (sobreinversión) ligadas a su propio funcionamiento. Así, estas teorías destacan la existencia de desajustes en el stock de capital y la demanda de consumo; de modo que debe modificarse la relación inversión y demanda total; para retornar a una situación de equilibrio.

La teoría del ciclo endógeno recibió un fuerte respaldo con el desarrollo de los modelos matemáticos del ciclo económico surgidos desde 1935. Estos modelos (nacidos al amparo de la tradición keynesiana) partían de un sistema dinámico de ecuaciones lineales que (sometido a perturbaciones endógenas) generaba ciclos en la Producción. Hasta 1965, las contribuciones a la teoría del ciclo endógeno partieron de los trabajos realizados por los keynesianos. Estos trabajos se agruparían en: modelos lineales de acelerador-multiplicador y modelos no-lineales deterministas.

\section{Una visión prospectiva de las teorías del ciclo económico permite clasificarlas en:}

\subsection{Teorías del ciclo real (Sargent y Wallace 1975; Kydland y Prescott, 1982)}

Son Teorías de estructuras de equilibrio, donde los ciclos reflejan reacciones de equilibrio de los agentes; que son consistentes con los supuestos de: vaciado continuo de los mercados, agente representativo optimizador y eficiencia del sistema económico. 


\section{Pensamiento Crítico Vol. 20. NoI}

Son teorías en que se verifica el supuesto de neutralidad del dinero a corto y largo plazo: los agentes (como los agentes pueden discriminar entre los diferentes tipos de perturbaciones que golpean al sistema económico y son capaces de anticiparse a sus efectos) sólo modifican sus decisiones óptimas de trabajo, producción y consumo en respuesta a perturbaciones de origen real (perturbaciones que causan cambios en los precios relativos).

Estas teorías consideran que el empleo, la producción y tecnología son variables reales, y que la oferta monetaria, precios e inflación son variables nominales. Las fluctuaciones de esas variables reales causan los ciclos económicos y las fluctuaciones de esas variables nominales son neutrales.

Estas teorías señalan que el alto costo de la mano de obra conduce a fuertes inversiones en tecnología (maquinaria y tecnología minera) y es la causa principal de los ciclos económicos. Los teóricos del Ciclo Real consideran que la tecnología tiende a crear más empleo indirecto que directo, originando modificaciones regionales y sectoriales del empleo.

Así, los teóricos del ciclo real analizaron: los efectos de los diferentes tipos de perturbaciones y los canales que permitían su transmisión; y el rol de los shocks tecnológicos como origen de los ciclos económicos.

En estas teorías, los ciclos son resultado de las reacciones de equilibrio de los agentes, quienes asignan: su tiempo entre trabajo y ocio, y su renta entre consumo presente y consumo futuro, en respuesta a alteraciones de la productividad, en un mundo de mercados competitivos y eficientes.

Dado que esas perturbaciones se producen por el lado de la oferta (quedando fuera del control del gobierno) el ajuste recaería directamente sobre el sector privado, de modo que la política económica debería limitarse a eliminar obstáculos para que trabajadores y empresarios pudiesen afrontar sus procesos de ajuste de la manera más flexible y menos dolorosa posible. 


\section{Pablo Rivas Santos}

Estas teorías se basan en modelos dinámicos de equilibrio general con mercados competitivos que se vacían continuamente a los precios de equilibrio, y se apoyan en una estructura de agente dotado de expectativas racionales que maximiza continuamente su función objetivo en un mundo de información perfecta. El supuesto de expectativas racionales implica que el agente conoce la estructura y el funcionamiento del sistema económico en el que debe tomar sus decisiones. El agente es capaz de anticipar los efectos de cualquier perturbación monetaria o fiscal (basada en reglas). El supuesto de información perfecta implica que el agente puede diferenciar entre perturbaciones de origen real y de origen monetario.

Esta teoría para la economía latinoamericana fue explicada por Robert Barro en su Macroeconomía $2^{\circ}$ edición 1994. Alianza Editorial y por Stephen Williamson en su Macroeconomía $4^{\circ}$ edición 2012. Editorial Pearson.

\subsection{Teorías del ciclo monetario}

Son Teorías que (al No Considerar el supuesto de mercado competitivo) No Aceptan el supuesto de neutralidad del dinero a corto plazo por lo que la Política monetaria expansiva aumenta la producción a corto plazo:

(a) Teorías keynesianas de mercados no competitivos. En estas teorías, la existencia de mercados no competitivos (estructuras monopolísticas u oligopolísticas) permiten a las empresas disponer de cierto control sobre sus precios (Blanchard, 1987).

(b) Teorías neokeynesianas de Rigideces de precios y salarios. En estas teorías los shocks de demanda (de origen monetario) no son neutrales a corto plazo como consecuencia de rigideces de precios y salarios atribuibles a:

- La presencia de "los costos de menú": los precios son rígidos a bajar, sí la empresa espera que los beneficios netos (derivados de esa baja) sean negativos (Mankiw, 1985).

- Los salarios son Rígidos a bajar como consecuencia decontratos salariales a largo plazo (Taylor, 1979). 


\section{Pensamiento Crítico Vol. 20. NoI}

Algunos neokeynesianos (Akerlof y Yellen, 1985) consideran que los ciclos económicos son causados por shocks aleatorios de innovación. Estos Neokeynesianos introducen el factor aleatorio (impredecible) de la innovación como un factor contemporáneo y novedoso de la causa de los Ciclos Económicos.

En a y b la existencia de esas barreras que dificultan el ajuste inmediato de precios y salarios, favorece que la política monetaria expansiva aumente la producción a corto plazo.

Son Teorías donde se supone la existencia de problemas de información imperfecta; por lo que la Política monetaria expansiva aumenta la producción a corto plazo como consecuencia de:

(a) Problemas de "ilusión monetaria" vinculados a la percepción de los salarios reales La teoría de Friedman de la curva de Phillips aumentada por las expectativas (Friedman, 1968) es una Teoría basada en el mercado de trabajo

- La política monetaria expansiva aumenta la producción real en el corto plazo, pero, en el largo plazo aumentará la producción nominal, los precios nominales, tasas de interés nominales, y esa producción real regresará a su nivel natural que depende de factores reales: supuesto de la neutralidad del dinero a largo plazo.

- Los salarios y precios muestran cierta lentitud de ajuste en el corto plazo (siendo más pronunciada en los salarios que en los precios) pero en el largo plazo alcanzarán su valor de equilibrio. Esa lentitud de ajuste de los salarios (respecto a los precios) crea el problema de "ilusión monetaria" en el corto plazo. En un entorno inflacionario donde los precios suben más rápido que los salarios, los trabajadores creerán que sus salarios nominales están subiendo -sin darse cuenta de que se está reduciendo el poder adquisitivo de sus salarios- $e$ incrementarán su oferta de trabajo. Pero, como los errores de percepción son temporales, los trabajadores darán marcha atrás en sus decisiones; anulando los efectos reales de corto plazo de la sorpresa monetaria (inflacionaria) inicial. 


\section{Pablo Rivas Santos}

- El dinero es el factor que causa los ciclos en la producción en torno a su valor natural (dados los efectos de corto y largo plazo de la Política monetaria expansiva). Por tanto, un uso discrecional de la política monetaria se convierte en la causa de inestabilidad de la producción real.

- Aceptar que existe una relación inversa entre inflación y desempleo que puede ser controlada en el largo plazo por los gobiernos equivale a aceptar que los trabajadores carecen de expectativas inflacionarias (incluso en un entorno de aumento continuo de precios).

Así, los trabajadores padecen un problema de "ilusión monetaria" que les impide percibir de manera inmediata el efecto de la inflación no esperada sobre sus salarios reales, pero esta situación no puede perdurar. Cuando los trabajadores descubren que los precios nominales suben más rápido que sus salarios nominales, modifican sus expectativas inflacionarias y negocian un incremento salarial que les permita recuperar su poder adquisitivo. Es en ese momento cuando desaparecen los efectos reales de las sorpresas monetarias inflacionarias; y la producción regresará a su nivel natural. En consecuencia, esa relación inversa inflación-desempleo sólo puede ser de corto plazo, desapareciendo en el largo plazo. La curva de Phillips de pendiente negativa se transforma en una curva vertical a la altura de la tasa de desempleo natural. Esta situación muestra la irrelevancia de las sorpresas monetarias (inflacionarias) para estimular la producción y el empleo a largo plazo: supuesto de neutralidad del dinero.

En un escenario donde los trabajadores están dotados de expectativas inflacionarias adaptativas, sí los gobiernos todavía intentaran controlar en el largo plazo ese trade-off inflación-desempleo, sólo podrían lograrlo mediante continuas aceleraciones no esperadas -cada una de ellas de efecto transitorio- de la inflación. Es decir, dado el supuesto de "ilusión monetaria" que caracteriza a los trabajadores; los gobiernos deberían sorprender a los trabajadores continuamente en sus expectativas inflacionarias mediante continuas aceleraciones dela inflación. Por ello, a la teoría de la tasa de desempleo natural friedmaniana se le llama hipótesis aceleracionista. 


\section{Pensamiento Crítico Vol. 20. NoI}

\section{El "Plucking Model" Friedmaniano}

Friedman en 1993 desarrolló una teoría del ciclo monetario alternativo a la teoría de la curva de Phillips aumentada por las expectativas. Según Friedman, las tasas de crecimiento de la economía norteamericana entre 1950-1992 apoyaban la existencia de un efecto "techo superior": las tasas de crecimiento eran inferiores al techo superior, pero tendían hacia el techo.

En ese modelo, un crecimiento de la economía de pleno empleo es interrumpido por un desequilibrio monetario: una contracción monetaria tira la economía hacia abajo situándola por debajo de su senda de crecimiento a largo plazo y provocando una recesión. Ese tirón se interpreta como error de política monetaria aleatorio que reduce la oferta monetaria o no la aumenta en respuesta a un aumento de la demanda de dinero. Ese exceso de demanda de dinero resultante junto con la rigidez mostrada en los precios y salarios genera una recesión; seguida por una recuperación posterior, que podría estar ayudada por la aplicación de políticas de demanda expansivas.

Los ciclos de la producción sólo se producen por debajo de esa senda de crecimiento a largo plazo, como consecuencia de rigideces institucionales.

(b) Problemas para extraer la señal contenida en los precios relativos (Lucas, 1972). La teoría con expectativas racionales de Lucas es una teoría del ciclo monetario basada en el mercado de productos

Lucas muestra que (en un mundo de información imperfecta) las variaciones monetarias no esperadas provocan ciclos temporales en la producción de los empresarios, como consecuencia de las dificultades para extraer la señal contenida en sus precios relativos: la expansión monetaria aumenta la producción a corto plazo como consecuencia de problemas de extracción de la señal contenida en los precios relativos.

Lucas desarrolló un modelo de equilibrio general donde todos los mercados se vacían continuamente y los empresarios optimizan sus funciones objetivo. Estos empresarios toman sus decisiones de producción en respuesta a variaciones de los precios relativos que resulta de perturbaciones reales (del mercado en el que operan) y 


\section{Pablo Rivas Santos}

no a variaciones del nivel general de precios que resulta de perturbaciones monetarias que afectan al conjunto de los mercados.

No obstante, estos empresarios padecen de un problema de información incompleta que les impide diferenciar si esas variaciones de los precios relativos son exclusivas del mercado en el que operan o responden a variaciones del nivel general de precios.

Esas variaciones monetarias no esperadas provocan ciclos temporales; sí esas variaciones del nivel general de precios se malinterpreta como variaciones de los precios relativos.

Sólo cuando los empresarios se den cuenta que el aumento de sus precios de producción, no ha sido exclusiva del mercado en el que operan, sino que responde a una variación monetaria, corregirán sus errores de decisión y la producción regresará a su nivel de equilibrio inicial.

Se dice que la teoría de Lucas adolece de un canal de transmisión que convierta esas variaciones monetarias no esperadas en ciclos permanentes.

Sí el problema de información incompleta se convierte en la causa de los ciclos, la sociedad obtendría un gran beneficio, haciendo pública esa información relevante, que induce a error a los agentes.

Esta teoría de las malas percepciones monetarias mostraría que usar el mecanismo de formación de expectativas racionales (alternativo al mecanismo aceleracionista friedmaniano) implicaría que el gobierno no podría hacer un uso continuo de la curva de Phillips para mantener el desempleo por debajo de su nivel natural.

Así, Lucas desarrolla una teoría en que las variaciones no esperadas de la cantidad de dinero generan alteraciones transitorias (en la misma dirección) de la actividad económica real por problemas de "extracción de la señal" contenida en los precios relativos. 


\section{Pensamiento Crítico Vol. 20. NoI}

Finalmente, Lucas considera que las familias y los empresarios predicen las políticas monetarias del BCR, por lo que las políticas monetarias expansivas sólo pueden ser eficientes si se toman por sorpresa. Las expectativas tienen un costo de información. Las expectativas de los empresarios e instituciones financieras son más eficientes y constituyen las expectativas racionales.

(c) Problema para extraer la señal contenida en las tasas de interés (Hayek, 1976).La teoría del ciclo monetario de Hayek es una teoría basada en el mercado de capitales.

Explica que los ciclos recurrentes de auge y recesión son resultado de Políticas Monetarias Expansivas ejecutadas por un sistema bancario de reserva fraccionaria. Esta expansión del crédito sin respaldo previo del ahorro, genera unas tasas de interés artificialmente bajas que descoordinan las decisiones intertemporales de productores $y$ consumidores, materializada en un exceso de inversión a largo plazo, que el mercado no será capaz de absorber. Por tanto, la estructura productiva sufre distorsiones que no responden a las disponibilidades de recursos ni a las preferencias de los consumidores; debiendo finalmente reajustarse a las preferencias de los consumidores.

Se asume un escenario donde el ahorro, consumo e inversión están alineados. Luego, se contrasta este escenario con las perturbaciones causadas por las Políticas Monetarias Expansivas.

\section{Auge sostenible: canal de transmisión}

Los bienes de capital presentan diferentes grados de heterogeneidad y complementariedad, siendo empleados en procesos de producción de duración variable para producir bienes de consumo.

Los empresarios emprenden proyectos de inversión de largo plazo sí estos proyectos son más productivos y rentables, Para producir más bienes de capital con los que emprender esos proyectos, se requiere de una mayor acumulación de ahorro con los que mantener a los factores de producción necesarios por más tiempo. A medida que aumenta el ahorro, la resultante estructura productiva se vuelve más intensiva en capital alcanzandoun mayor grado de desarrollo. 


\section{Pablo Rivas Santos}

Cuando aumenta el ahorro, la tasa de interés se reduce, lo que le señala a los empresarios que hay más recursos reales disponibles para emprender esos proyectos. Esa reducción de la tasa de interés aumenta el precio de los bienes de capital; dado que sus flujos de caja futuros se descuentan a esas tasas de interés más reducidas. Como consecuencia, aumenta la rentabilidad de invertir en proyectos de inversión más intensivos en capital; alargándose la estructura del proceso productivo.

Cuando aumenta el ahorro; los consumidores reducen su consumo presente (para incrementar su consumo futuro). En consecuencia, cae la rentabilidad de las etapas del proceso productivo destinados a producir bienes de consumo presente. Estas etapas, a su vez, comienzan a liberar recursos económicos que se destinan a la producción de bienes de capital cuyos precios aumentan -y por tanto la rentabilidad- como consecuencia de esa reducción de la tasa de interés. Así, estos cambios son causados por procesos microeconómicos

Como resultado de esos procesos microeconómicos (impulsados por empresarios que tratan de obtener beneficios) la estructura productiva se hace más alargada $e$ intensiva en capital, alcanzando un mayor grado de desarrollo. Los recursos liberados de esas etapas más próximas al consumo presente se emplean en las etapas más alejadas del consumo presente (donde se producen nuevos bienes de capital). Así, el orden espontáneo del mercado coordina las acciones de inversionistas y consumidores. Los consumidores ahorran más porque desean incrementar su consumo futuro. Al mismo tiempo, el aumento del ahorro permite alargar y ensanchar la estructura productiva para producir más bienes de consumo futuro. Finalizados esos nuevos proyectos, el consumo aumenta en términos reales ya que la producción es más intensiva en capital, algo que ha sido posible gracias a la acumulación de ahorro.

\section{Auge insostenible: canal de transmisión}

Las etapas del crecimiento insostenible se inician con una reducción de las tasas de interés. En este caso se trata de una reducción artificial, que no ofrece una señal fiable del verdadero ahorro que existe en el mercado.

Las políticas monetarias expansivas favorecen una reducción de las tasas de interés que no responde a una previa acumulación de ahorro. Sin embargo, dado que aumenta 


\section{Pensamiento Crítico Vol. 20. NoI}

la oferta de fondos prestables, su efecto es el mismo que si se hubiese producido la previa acumulación de ahorro. Por tanto, en un inicio, los escenarios de crecimiento sostenible y de crecimiento insostenible son idénticos: es decir, los precios de los bienes de capital tienden a subir y los nuevos proyectos parecen rentables como consecuencia de la reducción de las tasas de interés. En consecuencia, los empresarios alargan y ensanchan la estructura del proceso productivo, desplazando los factores de producción hacia esos nuevos proyectos.

Sin embargo, ese alargamiento y ensanchamiento no es sostenible ya que no se han liberado los recursos necesarios para mantener esos nuevos proyectos de inversión que son financiados con créditos de nueva creación que no están respaldados con ahorro. Por tanto, existe descoordinación entre los planes de decisión intertemporales de los agentes económicos: los empresarios (fijándose en unas tasas de interés artificialmente bajas) invierten como si el ahorro hubiese aumentado. Por el contrario, los planes de gasto de los consumidores siguen siendo los mismos de antes, de modo que no ahorran lo suficiente para sufragar esas nuevas inversiones. Esta descoordinación resulta insostenible y se revertirá tarde o temprano.

La reversión y la recesión. El alza de precios de los factores de producción es resultado de una demanda creciente de factores de producción para implementar esos nuevos proyectos; sin que estos factores hayan sido producidos previamente. Por consiguiente, los costos de esos nuevos proyectos comienzan a elevarse por encima del nivel esperado; no obstante, el optimismo de esa expansión del crédito y las nuevas inyecciones de dinero hacen que el auge continúe durante algún tiempo.

El aumento de precios de los bienes de consumo final se debe: a que los propietarios de factores de producción gastan el aumento observado en su renta, y que la producción de bienes de consumo final se reduce como consecuencia de la transferencia de factores de producción hacia etapas del proceso productivo más alejadas del consumo final.

Con ese aumento de precios de los bienes de consumo final, las ganancias de los proyectos de las etapas productivas más cercanas al consumo final se elevan respecto a las ganancias generadas por las etapas productivas más alejadas del consumo final. 


\section{Pablo Rivas Santos}

Además, las tasas de interés también se elevan, a medida que la expansión del crédito se reduce. Esta elevación de las tasas de interés provoca una caída de precios de los bienes de capital, haciendo que muchos proyectos dejen de ser rentables $e$ incluso deben abandonarse. Así, el período de auge artificial llega a su fin, cuando aparecen las pérdidas en las etapas productivas más alejadas del consumo final.

Ese alargamiento y ensanchamiento de la estructura productiva no es sostenible ya que esos nuevos proyectos se han financiado con créditos de nueva creación a una tasa de interés artificialmente baja; no existe un volumen de ahorro suficiente para mantener esos nuevos proyectos. Al contrario de los procesos de crecimiento sostenible (donde las nuevas inversiones son financiadas con acumulaciones de ahorro) la reducción artificial de las tasas de interés induce a error a los empresarios. Estos empresarios inician más proyectos de los que pueden sostenerse con el volumen de ahorro existente. Por consiguiente, durante la etapa recesiva, el mercado tenderá a depurar esas malas inversiones produciéndose una liberación de recursos. La recesión terminará cuando la estructura productiva se adapte nuevamente a los recursos reales disponibles en la economía y a las preferencias de los consumidores.

Una tasa de interés monetaria artificialmente baja crea una tendencia, para que el capital se reasigne de manera inconsistente con la tasa de interés de mercado y con las realidades económicas subyacentes a la tasa de interés de mercado (tecnología, disponibilidades de recursos, o preferencias de consumo de los agentes). Esa reasignación del capital causada por esa tasa de interés monetaria artificialmente baja, es la base del ciclo expansivo ficticio (burbuja) que contiene las semillas de su propia destrucción.

- La intervención del BCR en la fijación de la tasa de interés causa las recesiones, y la aplicación de políticas monetarias expansivas en esos casos, sólo posterga los ajustes necesarios.

- Esa fijación de la tasa de interés causa distorsiones en la estructura intertemporal de la producción al causar un desajuste en los planes de producción y de consumo, lo que finalmente se traduciría en un ajuste severo. 


\section{Pensamiento Crítico Vol. 20. NoI}

a) Características de la teoría del capital

a.1. El proceso de producción es una secuencia de etapas que requiere tiempo. Si el trabajo y los recursos naturales son los medios del proceso de producción, y los bienes de consumo son los fines del proceso de producción, entonces la estructura del capital ocupa una posición intermedia entre ambos, acentuando la dimensión intertemporal que caracteriza al proceso de producción. Así, el proceso de producción es una estructura por etapas que refleja: su carácter dinámico, intertemporal y la heterogeneidad del capital (donde el capital físico está compuesto por factores de producción altamente desagregado y especializado). Se destaca la naturaleza temporal del proceso de producción, sosteniendo que el proceso de producción añade valor a los factores de producción porque requiere tiempo.

a.2. Los precios y las tasas de interés (en un entorno de información imperfecta) actúan como señales. Transmiten información que guía las decisiones de producción y de consumo de los agentes. Reflejan: los cambios en las valoraciones subjetivas de los consumidores, y la escasez relativa de los recursos alternativos. Es una red de comunicación fiable que establece la base para una coordinación económica eficaz.

Por tanto, si las señales contenidas en los precios son falseadas por las perturbaciones monetarias, se produce una descoordinación insostenible entre los planes de producción y de consumo de los agentes.

a.3. Cuando el BCR expande la cantidad de dinero inyectándola a través del sistema del crédito, ese dinero se oculta como ahorro y produce una diferencia entre el ahorro y la inversión.

Una tasa de interés artificialmente baja, estimula a los empresarios a endeudarse más para invertir, mientras que los consumidores prefieren ahorrar menos. Por tanto, provocará que la inversión sea inconsistente con la cantidad de recursos (el ahorro) generada en la economía; y con las preferencias de consumo intertemporales de los consumidores. 


\section{Pablo Rivas Santos}

a.4. Los bienes de capital de orden superior (que se emplean en las primeras etapas del proceso de producción más alejadas del consumo final) y los bienes de capital de orden inferior (que se emplean en las últimas etapas del proceso de producción más cercanas al consumo final) son complementarios intertemporalmente.

Así, esa descoordinación intertemporal (impulsada por esa tasa de interés artificialmente baja) se manifiesta a través de la sobreinversión en bienes de capital de orden superior, y provoca una sustitución de bienes de capital de orden inferior por bienes de capital de orden superior. No obstante, el transcurrir del tiempo y la escasez de bienes de capital de orden inferior, revelará la existencia de esa descoordinación intertemporal, insalvable, sin el ajuste necesario contenido en un proceso recesivo.

Los bienes de capital son heterogéneos y reflejan complementariedad y sustituibilidad intertemporal

a.5. Una reducción del consumo presente señala un aumento del consumo futuro, que desplazará los bienes de capital de orden inferior que se emplean en las últimas etapas del proceso de producción (orientadas al consumo presente) hacia las primeras etapas del proceso de producción (orientadas al consumo futuro).

a.6. Hay dos tipos de conocimiento: conocimiento (vinculado a circunstancias particulares de tiempo y lugar) que poseen los agentes a través de su propia experiencia, y conocimiento científico de cuál es la estructura y el funcionamiento del sistema económico en el que los agentes deben tomar sus decisiones.

Dado que los agentes disponen del primer tipo de conocimiento pero no disponen del segundo tipo de conocimiento, no serán capaces de discernir entre: alteraciones de precios debidos a perturbaciones monetarias o reales. La perturbación monetaria induce a error a los agentes, incitándoles a comportarse de manera diferente a como lo hubiesen hecho en ausencia de la perturbación monetaria. Así, los agentes toman sus decisiones en un entorno de conocimiento imperfecto. 


\section{Pensamiento Crítico Vol. 20. Nº}

Los empresarios toman decisiones: sobre la base de su propia experiencia (conocimiento práctico) y de su expectativa, e informados y coordinados por los movimientos de precios, salarios y tasas de interés. Esas decisiones empresariales causan una asignación intertemporal del capital, que puede ser sostenible (internamente consistente) o insostenible (que pueda implicar alguna inconsistencia interna sistemática que transforma cualquier proceso expansivo en una recesión).

Así, la teoría del capital estudia los factores que hacen que un proceso de auge sea sostenible o insostenible (que contiene las semillas de su propia destrucción).

\section{b) Mercado de Capitales y Ciclo Económico}

b.1. La demanda de fondos prestables refleja la disposición de los empresarios a participar en el proceso de producción recurriendo al endeudamiento.

La oferta de fondos prestables responde a los consumidores que ofrecen su ahorro (renunciando al consumo presente) a cambio de una rentabilidad.

El mercado de fondos prestables facilita la coordinación de los planes de producción de los empresarios con las preferencias intertemporal de los consumidores.

El precio que equilibra el mercado de fondos prestables (haciendo que la asignación de recursos sea internamente consistente) es la tasa de interés de mercado i* que representa la tasa de sustitución intertemporal de los participantes del mercado.

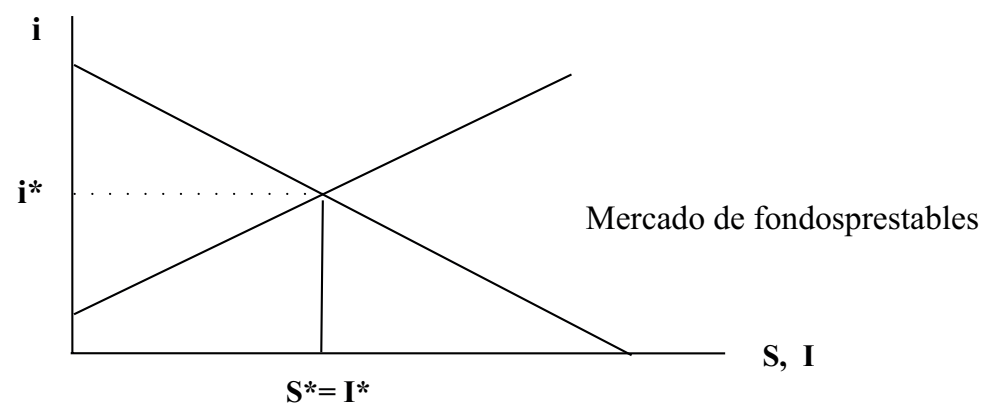




\section{Pablo Rivas Santos}

Esa tasa de interés i* coordina los planes de producción de los empresarios con las preferencias intertemporales de los consumidores.

b.2. La Frontera de Posibilidades de Producción FPP representa el trade-off entre bienes de consumo y bienes de capital. Cualquier punto situado en la FPP muestra una situación de pleno empleo y refleja combinaciones de consumo e inversión sostenibles. Puntos por debajo de la FPP representa una situación de desempleo temporal de los recursos (desempleo involuntario) y puntos por encima de la FPP representa una situación de sobreempleo temporal de los recursos.

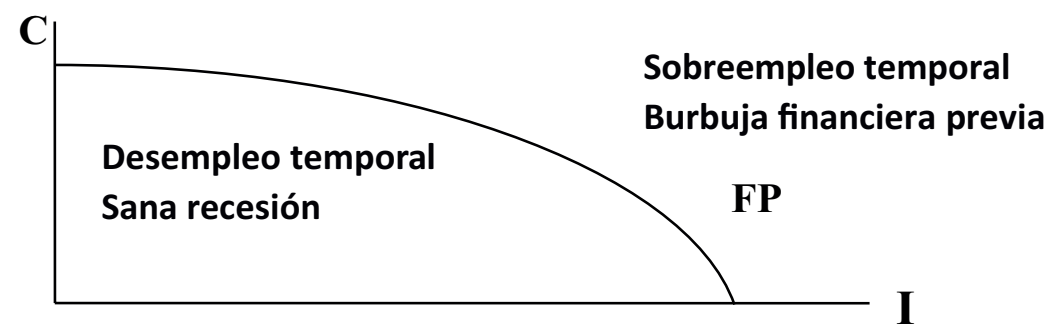

b.3. La estructura intertemporal de la producción refleja los dos aspectos de la teoría del capital $1 .^{\circ}$ La producción es un proceso que requiere tiempo, $2 .^{\circ}$ Este proceso es una secuencia de etapas (que caracteriza la estructura intertemporal del capital).

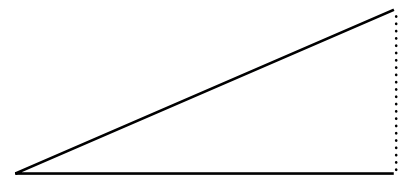

Primeras Etapas de la producción Ultimas Etapas de la producción

El cateto horizontal representa el tiempo de la producción (que implica la secuencia de etapas de la producción) refleja la dimensión temporal (que caracteriza al proceso de producción).

El cateto vertical representa el valor monetario de la producción que (en equilibrio) coincide con el valor monetario del consumo. 


\section{Pensamiento Crítico Vol. 20. NoI}

La hipotenusa representa el valor monetario de los bienes intermedios y su pendiente refleja la tasa de rentabilidad implícita entre las diferentes etapas del proceso de producción.

Los factores de producción de la primera etapa del proceso de producción (más alejada del cateto vertical) se emplean para producir bienes de consumo futuro.

Los factores de producción de la última etapa del proceso de producción (más cercana al cateto vertical) se emplean para producir bienes de consumo presente.

Estas etapas del proceso de producción cambian en respuesta a cambios en la tasa de interés que equilibra el mercado de fondos prestables: una baja de la tasa de interés estimula la inversión en la primera etapa del proceso de producción (más intensiva en capital) en detrimento de la última etapa.

La interrelación de esas representaciones b.1, b.2, b.3 permite analizar y diferenciar los aspectos relacionados al Auge sostenible (vinculado a aumentos del ahorro, mejoras tecnológicas y las preferencias por la liquidez de los agentes) y al Auge insostenible (inducido políticamente).

Así, el grado de coordinación entre las preferencias intertemporales de los consumidores y los planes de producción de los empresarios, determinará sí el proceso de mercado (que asigna los recursos a lo largo del tiempo) es sostenible o insostenible.

\section{c) Auge sostenible versus Auge insostenible}

El Auge sostenible ocurre cuando las decisiones de inversión (en nuevo stock de capital) son compatibles con las disponibilidades de recursos (ahorro) y las preferencias intertemporales de los consumidores. Sí el BCR mantiene una política de estabilidad monetaria, entonces los incrementos de productividad se traducirán en una baja de precios y un aumento de salarios reales.

El Auge insostenible ocurre cuando las decisiones de inversión son incompatibles con las disponibilidades de recursos y las preferencias intertemporales de los consumidores. 


\section{Pablo Rivas Santos}

El criterio para diferenciar los procesos de Auge sostenible e insostenible es la tasa de interés determinada en el mercado de fondos prestables que permite a la economía crecer a una tasa sostenible.

Establecer una tasa de interés artificialmente baja (inferior a esa tasa de interés de mercado) destinada a estimular el Auge, provocará un enfrentamiento por los recursos financieros entre ahorristas e inversionistas, situando a la economía en una senda de Auge insostenible.

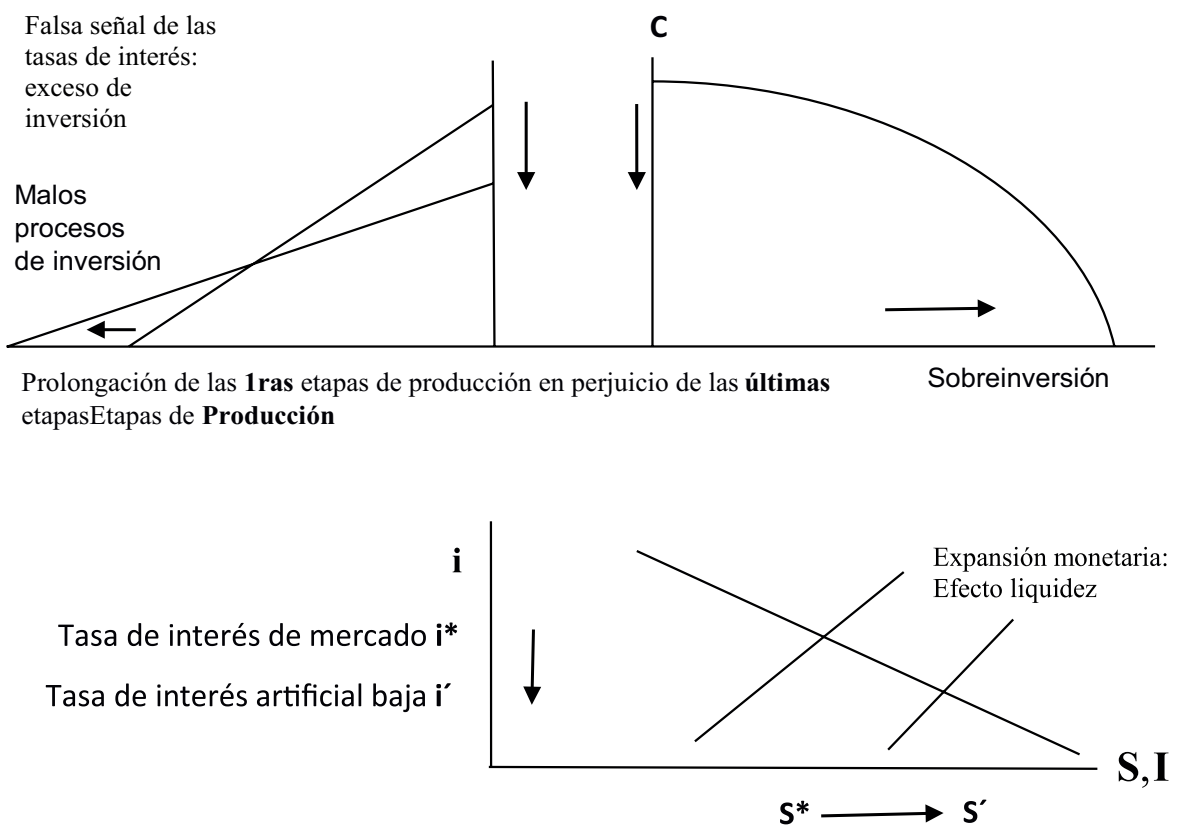

El proceso del Auge sostenible se vincula al aumento del ahorro que generará el efecto demanda derivada (que supone una reducción de la demanda de bienes de consumo presente) que reducirá la producción de bienes de consumo presente y la producción de bienes intermedios (que son necesarios para producir esos bienes de consumo presente) causando un efecto recesivo en todas las etapas del proceso de producción. 


\section{Pensamiento Crítico Vol. 20. NoI}

Pero, ese efecto recesivo de la demanda derivada es amortiguado por el efecto descuento temporal, porque los individuos ahorran con una finalidad: tratan de incrementar su consumo futuro prestando dinero a cambio de una rentabilidad, un aumento de la oferta de fondos prestables baja la tasa de interés (como consecuencia del efecto liquidez).

Esa baja de la tasa de interés genera un efecto descuento temporal: efecto que es muy débil en las últimas etapas del proceso de producción, y aumenta a medida que nos desplazamos hacia las primeras etapas del proceso de producción. En esas primeras etapas del proceso de producción prevalece el efecto expansivo del descuento temporal sobre el efecto recesivo de la demanda derivada.

La alteración que se produce en la forma del triángulo: muestra una acumulación de la inversión en esas primeras etapas del proceso de producción (orientada a la producción de bienes de consumo futuro) en detrimento de esas últimas etapas del proceso de producción (orientada a la producción de bienes de consumo presente). Por otro lado, la nueva pendiente de la hipotenusa del triángulo es menor en consonancia con esa menor tasa de interés.

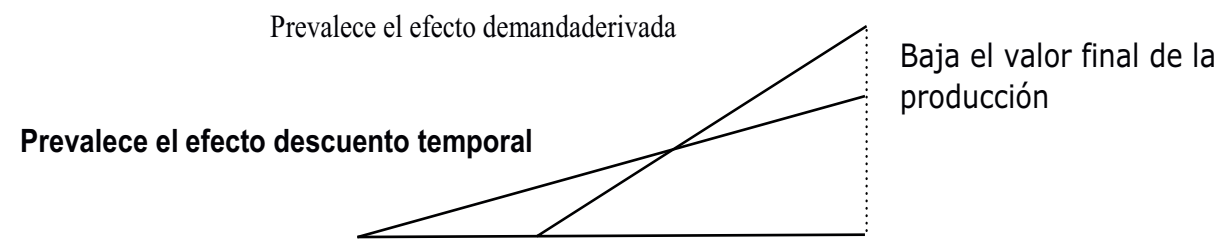

Aumenta la inversión en las primerasetapas

Esa menor pendiente de la hipotenusa del triángulo coincide con la menor tasa de interés que equilibra el mercado de fondos prestables: la tasa de interés implícita (en las diferentes etapas del proceso productivo) se iguala a la tasa de interés de mercado (la tasa que representa la tasa de sustitución intertemporal de los consumidores-ahorristas). Este proceso es, el que coordina las decisiones intertemporales de productores y consumidores haciendo que sean mutuamente compatibles. 

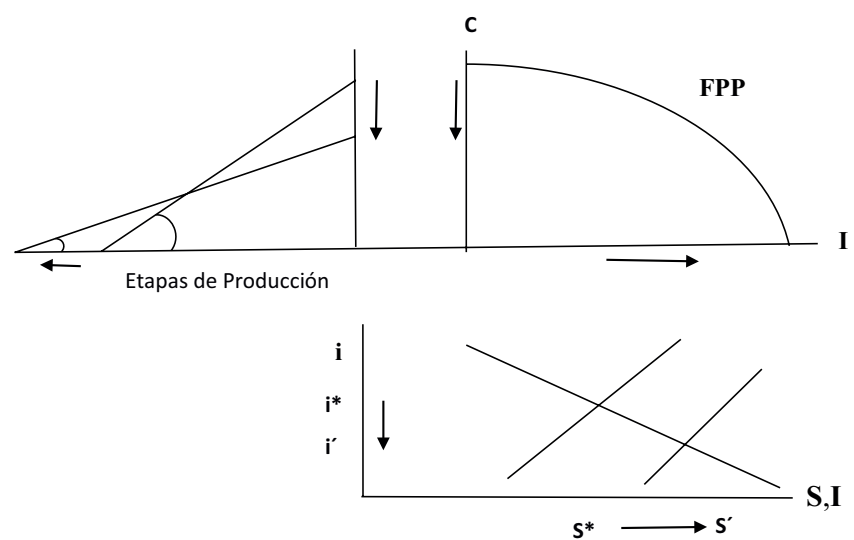

Fuente: El triángulo de Hayek en Teoría Monetaria y el ciclo económico (1976)

Esa menor tasa de interés ofrece una señal fiable que orienta la asignación intertemporal de los factores de producción, de manera que sea coherente con los nuevos gastos (más orientados hacia el futuro) de los consumidores. El proceso de auge iniciado (apoyado en aumentos de la inversión, financiados con ahorro previamente generado) es sostenible, y se materializará en sucesivos desplazamientos en paralelo y hacia arriba del triángulo y de la FPP.

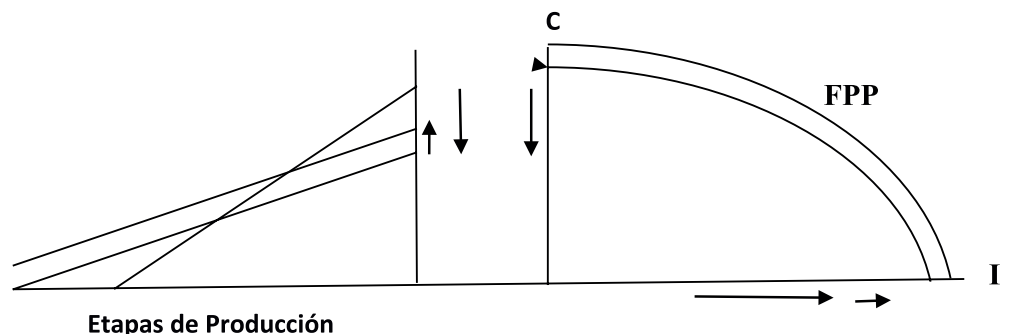

Etapas de Producción

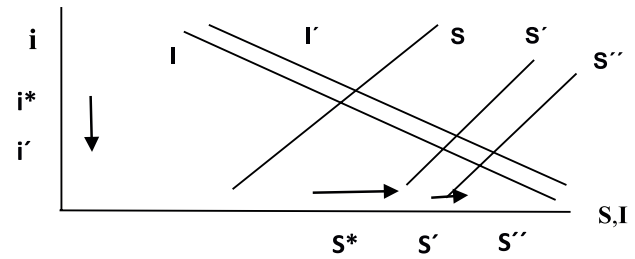




\section{Pensamiento Crítico Vol. 20. NI}

Así, consideramos que el marco teórico de la Incidencia de la Política Monetaria del BCR son la Teoría y Política Monetaria y el Ciclo Económico de John Maynard Keynes, Milton Friedman, Robert Lucas y Friedrich Hayek las cuales especifican las Causales Monetarias y los Mecanismos de Transmisión.

\section{Conclusiones}

La desventaja de usar el agregado macroeconómico Producción Nacionales que este oculta los mecanismos de cambio que ocurren dentro de la Producción Nacional. En la teoría de Keynes sólo interesa la relación entre los agregados macroeconómicos (producción, inversión, empleo) y no los movimientos dentro de la producción, inversión y empleo nacional. Esta crítica al excesivo agregacionismo keynesiano, se hace extensible a las teorías de Friedman y Lucas.

Las 4 teorías del ciclo monetario coinciden en caracterizar el ciclo económico como un problema de: malas percepciones y errores de decisión surgidos en un mundo de información imperfecta. El origen de esas malas percepciones y errores de decisión es la perturbación monetaria que reduce la eficiencia del sistema de precios como mecanismo asignador, señalizador y coordinador de la actividad económica.

Se admite, que el dinero perturba la producción(al menos en el corto plazo) siendo este un elemento que diferencia esas 4 teorías delas teorías del ciclo real.

Las teorías del ciclo real toman las relaciones de equilibrio como continuas restricciones vinculantes durante el transcurso del ciclo económico: definen los ciclos económicos como sucesiones continuas de precios y cantidades de equilibrio que varían conforme lo hace el entorno económico.

La teoría de Hayek define los ciclos económicos como casos de desequilibrio intertemporal sistemático.

Esta diferente caracterización de los ciclos económicos, identifica a esas 4 teorías como teorías del desequilibrio (o de los fallos de coordinación inducidos) frente a esas teoríasdel ciclo Real. 


\section{Pablo Rivas Santos}

Pero, en esas 4 teorías se reconoce que (en ausencia de políticas que interfieran en el libre funcionamiento del mercado) se impondrán las fuerzas equilibradoras del mercado.

Las teorías de Lucas y Hayek comparten la idea de la racionalidad en la acción humana, y recurren al individualismo metodológico que conlleva a una microfundamentación de la macroeconomía.

Pero, la teoría de Lucas defiende los postulados de homogeneidad y objetividad propio del agente representativo (empresario, trabajador consumidor) y la teoría de Hayek defiende los postulados de heterogeneidad y subjetividad de la información, el conocimiento y las expectativas de los individuos. Por tanto, el individualismo metodológico objetivista frente a individualismo metodológico subjetivista.

Las diferencias entre esas cuatro teorías se clasifican en términos: del método y objetivo, de la forma de caracterizar la economía y las perturbaciones monetarias, y de los factores que inciden en la transmisión y persistencia del ciclo económico.

a. En cuanto al método y objetivo, la teoría de Hayek explica el ciclo económico en términos de una multitud de planes individuales (de producción y consumo) parcialmente conflictivos o descoordinados, La teoría de Lucas intenta predecir el comportamiento del agente representativo individual, en el transcurso del ciclo económico.

b. En cuanto a la forma de caracterizar la economía, las teorías de Friedman y Lucas presentan una macroeconomía: agregada que oculta los mecanismos de cambio que se generan dentro de la Producción Nacional y basada en el factor trabajo. La teoría de Hayek presenta una macroeconomía desagregada que concede protagonismo: a la estructura intertemporal del capital, y a la heterogeneidad de los agentes. Define el proceso de producción como una estructura por etapas. Y junto con el énfasis en el mercado de bienes de capital, el carácter subjetivo de la información y de las expectativas en un mundo de información imperfecta, y la precisión con que las tasas de interés ejercen su función señalizadora/coordinadora son aspectos que diferencian los procesos de Auge sostenible, de los procesos que (inducidos políticamente) distorsionan la estructura del proceso de producción generando una descoordinación insostenible entre los planes de producción y de consumo. 


\section{Pensamiento Crítico Vol. 20. NoI}

Estos aspectos en la forma de caracterizar la economía, generan a su vez otras diferencias entre las teorías de Friedman, Lucas y Hayek.

Las teorías de Friedman y Lucas ignoran los efectos a corto plazo de la Política Monetaria expansiva sobre el mercado de bienes de capital, centrándose en esos efectos sobre el mercado de trabajo. Esto implica que (en un mundo de información imperfecta) el origen del ciclo económico debe buscarse en el hecho de que la expansión monetaria no esperada induce a los trabajadores a sobrevalorar sus salarios reales en la teoría de Friedman o induce a los empresarios a sobrevalorar los precios relativos a los que venden su producción en la teoría de Lucas impulsándoles a ofrecer más trabajo del que hubiesen brindado en otras circunstancias.

La orientación de la teoría de Hayek hacia el mercado de bienes de capital denota que el origen del ciclo económico está en el hecho de que la Política Monetaria expansiva(al falsear la información coordinadora contenida en las tasas de interés) induce a los empresarios a cometer errores de sobreproducción, observándose una reestructuración del proceso productivo incompatible con las disponibilidades de recursos y las preferencias de consumo intertemporales de los consumidores.

En las teorías de Friedman y Lucas: el excesivo agregacionismo con que se afronta el estudio del proceso de producción, y el recurso al helicóptero friedmaniano para caracterizar la Política Monetaria expansiva neutral; impiden ver el efecto de la política monetaria expansiva sobre la estructura del proceso de producción (efecto Cantillón) dependiendo de la forma en que se expande (a través del mercado de crédito).

c. En las teorías de Friedman y Lucas el mecanismo de Transmisión del ciclo económico es el efecto sustitución intertemporal en la relación trabajo-ocio, y en la teoría de Hayek el mecanismo de transmisión del ciclo económico es el efecto sustitución intertemporal entre las etapas que integran la estructura intertemporal de capital del proceso de producción.

A pesar que las teorías de Friedman y Lucas han ignorado la importancia de la estructura intertemporal del capital (desechando cualquier problema de descoordinación entre los planes de producción y de consumo de los agentes) la teoría de expectativas racionales explica la persistencia de los ciclos económicos suponiendo que las empresas 


\section{Pablo Rivas Santos}

"necesitan tiempo" para construir nuevo stock de capital. Así, Kydland y Prescott (1982) dicen que las decisiones de iniciar un proceso de producción por etapas inducida por la Política Monetaria expansiva, influyen en las acciones que deben tomarse en períodos futuros. Por tanto, esa teoría de "tiempo para construir" conduce a reintroducir el capital en su dimensión intertemporal dentro de la Macroeconomía.

Aunque las cuatro teorías del ciclo monetario coinciden en definir la fase expansiva como etapa de recalentamiento (donde existe sobreempleo temporal de los factores de producción) la fase recesiva se caracteriza por presentar un perfil diferente según la teoría en la que nos encontremos.

En las teorías de Friedman y Lucas, cuando los agentes se dan cuenta de los errores cometidos en la valoración de sus salarios/ingresos reales (de manera que la fase expansiva se convierte en fase recesiva) la economía regresará a sus niveles de producción y empleo naturales (en la FPP). Esto, les permite concluir que el dinero es neutral a largo plazo.

En la teoría de Hayek, la fase recesiva se caracteriza por eliminar los malos procesos de inversión desarrollados durante la fase de "la burbuja financiera" previa. Así, durante el período de reestructuración del proceso de producción la economía se situará por debajo de su nivel de producción potencial (de la FPP) observándose un subempleo de los factores de producción.

Las teorías de Friedman y Lucas sostienen que los errores de decisión repetitivos de los agentes (respecto a cuánto trabajar/producir) son consecuencia de la expansión monetaria no esperada, la teoría de expectativas racionales sostiene que cuando se hace un uso reiterado de esa sorpresa monetaria, ésta puede ser anticipada anulando sus efectos reales. El hecho de que aumente la cantidad de dinero (aunque sea de forma no esperada) no impide que los empresarios juzguen correctamente qué influencia ejercerá sobre los precios y las tasas de interés de mercado.

Puede ser que los empresarios reaccionen en el futuro (ante una expansión monetaria) de manera diferente a como reaccionaron en el pasado: puede ser que eviten utilizar el dinero fácil disponible de una expansión, en sus operaciones, porque mantendrán en mente el fin inevitable del auge. 


\section{Pensamiento Crítico Vol. 20. Nº}

Por tanto, esta teoría de expectativas racionales supone un reto al intento de dar una explicación monetaria a los ciclos económicos repetitivos. Un "ciclo económico anticipadosería un ciclo económico evitado". Pero, el ciclo económico sería inevitable cuando los empresarios utilizan el dinero fiduciario adicional. Esa Política Monetaria expansiva afectaría a las decisiones de los agentes, originando fases de descoordinación recurrentes entre las decisiones intertemporales de producción y de consumo.

\section{Referencias bibliográficas}

1. Alonso Neira, Miguel A. Las teorías del ciclo en el marco de la literatura sobre los ciclos económicos. Argentina. Revista Libertas XII (Octubre 2005)

2. Alonso Neira, Miguel A, Bagus Philipp, Rallo Julián, Juan Ramón.Teorías del ciclo económico: principales contribuciones y análisis a la luz de las aportaciones de la escuela austriaca de economía. Argentina. ICE (Enero-Febrero 2011)

3. Barro, Robert. MACROECONOMÍA. ESPAÑA. Alianza Editorial Pag. $70-85$ (1995)

4. Friedman, Milton. THE PLUCKING MODEL OF BUSINESS FLUCTUATIONS REVISITED. EEUU. Economic Inquiry, volumen 31 número 2, paginas 171-1767. (1993).

5. Friedman, Milton. NUEVA REFORMULACIÓN DE LA TEORÍA CUANTITATIVA DEL DINERO. ESPAÑA. Editorial Continental. Primera Edición. Pag. 123-152. (1971).

6. Lucas, Robert. TEORÍA DE LOS CICLOS ECONÓMICOS. ESPAÑA. Alianza editorial. Pag. 113-152. (1987).

7. Hamann Salcedo, Franz POLÍTICA MONETARIA Y LOS CICLOS ECONÓMICOS. COLOMBIA. Editorial Norma. Pag 24- 44 (2012)

8. Mundell, Robert A. TEORÍA MONETARIA. ESPAÑA. Ediciones Amorrurtu Editores. Pag. 323-430. (1971). 


\section{Pablo Rivas Santos}

9. Von Hayek Friedrich. DESNACIONALIZACIÓN DEL DINERO. ESPAÑA. Editorial Continental. Pag. 143-187. (1978).

10. Von Mises Ludwig. TEORÍA DE LA MONEDA Y EL CRÉDITO. ESPAÑA. Fundación Ignacio Villalonga. Pag. 239-452. (1966).

11. Von Mises Ludwig. ACCIÓN HUMANA. ESPAÑA. Fundación Ignacio Villalonga. Pag. 180-332. (1980).

12. Williamson, Stephen MACROECONOMÍA. ESPAÑA. Editorial Pearson.Pag. 20-60 (2012) 